\title{
Grenzen der Privatisierung öffentlicher Aufgaben aus verfassungsrechtlicher Sicht
}

Seit mehr als zwei Jahrzehnten werden in Deutschland immer mehr Bereiche der öffentlichen Daseinsvorsorge privatisiert und damit dem direkten Zugriff des Staates entzogen. Mittlerweile werden die Folgen dieser Entwicklung für die Bürger und das Gemeinwohl äußerst kritisch diskutiert. Bislang kaum öffentlich debattiert wird jedoch die Frage, inwieweit die umfangreichen Privatisierungsmaßnahmen überhaupt durch das Grundgesetz gedeckt sind. Das ist umso erstaunlicher, als bei einigen zentralen Privatisierungsprojekten, wie z. B. der Bahn und der Post, zuvor sogar ausdrücklich eine Änderung des Grundgesetzes vorgenommen wurde und vorgenommen werden musste, da diese Bereiche ursprünglich durch den Art. 87 Abs. 1 GG als Teil der öffentlichen Verwaltung definiert waren. Im folgenden Beitrag werden aus verfassungsrechtlicher Sicht die Grenzen der Privatisierung öffentlicher Aufgaben aufgezeigt und Ansätze für eine Rückgewinnung staatlicher Steuerungsfähigkeit diskutiert.

\section{DIE BEDEUTUNG DER ÖFFENTLICHEN DASEINSVORSORGE}

Mit der öffentlichen Daseinvorsorge sollen wichtige Infrastrukturbereiche bereitgestellt werden, die zur Sicherung eines menschenwürdigen Daseins notwendig sind. Hierzu gehören Leistungen bzw. Einrichtungen, derer der Mensch zur Verwirklichung seiner Person und Individualität bedarf und die er nicht selbst zur Verfügung stellen kann, wie z. B. Elektrizität, Wasserversorgung, Telefon, Straßen, Bahn und Post usw. Mit der universellen Bereitstellung öffentlicher Güter soll zudem gewährleistet werden, dass alle Menschen, unabhängig von der sozialen Stellung des Einzelnen, gleiche Chancen für die persönliche Entfaltung haben.
Aus der Perspektive des Grundgesetzes bildet die öffentliche Daseinsvorsorge ein zentrales Instrument, um durch die Verfassung vorgegebene gesellschaftspolitische Ziele zu erreichen. Hierzu gehört vor allem die Wahrung der Menschenwürde (Art.1 GG) und das Sozialstaatsprinzip (Art. 20 GG). Das Bundesverfassungsgericht hat in seiner Rechtsprechung immer wieder darauf hingewiesen, dass der Staat die Pflicht hat, für eine gerechte Sozialordnung zu sorgen (BVerfGE 59, $231<263>$; s. a. BVerfGE $82,60<80>$; 22, 180). Eine weitgehende Privatisierung der öffentlichen Daseinsvorsorge, die die Bereitstellung öffentlicher Güter den ungezügelten und nicht kontrollierbaren Interessen an Gewinnmaximierung unterordnet, läuft dieser staatlichen Verantwortung zuwider.

\section{DIE BEDEUTUNG DER MENSCHENWÜRDE}

Das Bundesverfassungsgericht hat schon in einer sehr frühen Entscheidung darauf hingewiesen (BVerfGE 6, $32<40>-$ Elfes -), dass das Grundgesetz eine wertgebundene Ordnung institutionalisiert hat, die die öffentliche Gewalt begrenzt. Durch diese Ordnung sollen die Eigenständigkeit, die Selbstverantwortlichkeit und die Würde des Menschen in der staatlichen Gemeinschaft gesichert werden (BVerfGE 2, 1 $<12$ f. $>$; $5,85<204$ ff. $>$ ). Hiermit wurden die Grundzüge des im Grundgesetz angelegten Menschenbildes definiert, die später um einen zusätzlichen Aspekt erweitert wurden (BVerfGE 7, $198<205>-$ Lüth -). Demnach hat das Grundgesetz in seinem Grundrechtsabschnitt eine objektive Wertordnung errichtet, die ein Wertesystem begründet, das innerhalb der sozialen Gemeinschaft die sich frei entfaltende menschliche Persönlichkeit und ihre Würde als
Mittelpunkt hat. Hiermit wurde eine verfassungsrechtliche Grundentscheidung getroffen, die für alle Bereiche des Rechts gelten muss (bestätigt etwa in BVerfGE 21, $362<372>$ ).

Im Hinblick auf die öffentliche Daseinsvorsorge hat das Bundesverfassungsgericht die im Art. 1 GG garantierte Menschenwürde unmittelbar in den Mittelpunkt seiner Betrachtung gestellt. So hat es befunden, dass z. B. die Energieversorgung zum Bereich der Daseinsvorsorge gehört (BVerfGE 66, $248<258>$ ). Sie sei eine Leistung, deren der Bürger zur Sicherung einer menschenwürdigen Existenz unumgänglich bedürfe. Schon in einer früheren Entscheidung (BVerfGE 38, $258<270 \mathrm{f}$. $>$ ) hat das Bundesverfassungsgericht darauf hingewiesen, dass es eine Entwicklung gibt, in deren Verlauf die öffentliche Hand in wachsendem Umfang im Bereich der Daseinsvorsorge Aufgaben übernimmt, die unmittelbar oder mittelbar der persönlichen Lebensbewältigung des einzelnen Bürgers dienen (hierzu auch BVerfGE 45, 63 $<78 \mathrm{f}$. $>$ ). Angesichts der fortschreitenden Privatisierung ist der Staat jedoch immer weniger in der Lage, die Rahmenbedingungen für ein dem Grundgesetz entsprechendes Menschenbild zu gewährleisten.

\section{DAS SOZIALSTAATSPRINZIP}

Nach dem Grundgesetz ist der Staat verfassungsrechtlich zu sozialer Aktivität verpflichtet, um einen erträglichen Ausgleich der widerstreitenden Interessen und die

Siegfried Broß, Prof. Dr., Richter am Bundesverfassungsgericht, Honorarprofessor an der Universität Freiburg, Vorsitzender des Präsidiums der Deutschen Sektion der Internationalen Juristen-Kommission. 
Herstellung verträglicher Lebensbedingungen für alle zu gewährleisten (BVerfGE $1,97<105>$ ). Das Sozialstaatsprinzip verpflichtet den Staat, für eine gerechte Sozialordnung zu sorgen (BVerfGE 22, 180). Damit nimmt das Bundesverfassungsgericht schon im Jahre 1967 das vorweg, was heute als Social Equity bezeichnet wird.

In der weiteren Rechtsprechung des Bundesverfassungsgerichts wird der Leistungsanspruch des Einzelnen gegen den Staat weiter präzisiert. Je stärker der moderne Staat sich der sozialen Sicherung und kulturellen Förderung der Bürger zuwendet, desto mehr tritt im Verhältnis zwischen Bürger und Staat neben das ursprüngliche Postulat grundrechtlicher Freiheitssicherung vor dem Staat die komplementäre Forderung nach grundrechtlicher Verbürgung der Teilhabe an staatlichen Leistungen (BVerfGE 33, $303<330 \mathrm{f}$. $>$; bestätigt etwa in BVerfGE $35,79<115>$ ).

Für die Gestaltung der Wirtschaftsordnung im Besonderen zieht das Bundesverfassungsgericht folgenden Schluss (BVerfGE 50, $290<338>-$ Mitbestimmung): Das Grundgesetz sei wirtschaftspolitisch neutral. Der Gesetzgeber dürfe jede ihm sachgemäß erscheinende Wirtschaftspolitik verfolgen, sofern er dabei das Grundgesetz, vor allem die Grundrechte, beachtet. Ihm komme also eine weitgehende Gestaltungsfreiheit zu. Allerdings dürfe die Berücksichtigung der Gestaltungsfreiheit des Gesetzgebers nicht zu einer Verkürzung dessen führen, was die Verfassung in allem Wandel unverändert gewährleisten will, namentlich dürfe es nicht zu einer Beschneidung der in den Einzelgrundrechten garantierten individuellen Freiheiten kommen, ohne die nach der Konzeption des Grundgesetzes ein Leben in menschlicher Würde nicht möglich ist. Die Aufgabe besteht infolgedessen darin, die grundsätzliche Freiheit wirtschafts- und sozialpolitischer Gestaltung, die dem Gesetzgeber gewahrt bleiben müsse, mit dem Freiheitsschutz zu vereinen, auf den der Einzelne gerade auch dem Gesetzgeber gegenüber einen verfassungsrechtlichen Anspruch habe (hierzu auch BVerfGE 7, $377<400>-$ Apotheken-Urteil -; s.a. auch BVerfGE 22, 180).

Zunehmend gewinnt in der Entwicklung der Rechtsprechung der Anspruchsoder Teilhabeaspekt an Gewicht, und daraus ergeben sich Verpflichtungen für die Ausgestaltung der Wirtschaftsordnung. Entscheidend ist in diesem Zusammen- hang die Verbindung zwischen den Grundrechten, vor allem der Menschenwürde des Art. 1 Abs. 1 Satz 1 GG und der Handlungsfreiheit des Art. 2 Abs. 1 GG mit dem Sozialstaatsprinzip des Art. 20 Abs. 1 GG. Das lässt den Schluss zu, dass nach dem Grundgesetz der Bundesrepublik Deutschland der Einzelne zwar eigenständig und selbstverantwortlich ist, der Staat ihn aber nicht sich selbst überlassen darf. Vielmehr ist dieser gehalten, verlässliche und gemeinverträgliche Grundlagen sicherzustellen, damit Rahmenbedingungen geschaffen und fortwährend aufrechterhalten werden, die eine friedliche Gesellschaft und die Interessen aller Menschen innerhalb dieser Gesellschaft angemessen berücksichtigen. Keinesfalls dürfen staatliche und wirtschaftliche Ordnung so gestaltet werden, dass die Gesellschaft auseinanderbricht und nur noch ein Teil gleichsam auf der Sonnenseite des Lebens steht. Es liegt auf der Hand, dass das Streben nach Gewinnmaximierung einem solchen Verständnis entgegensteht.

Da bei der Privatisierung öffentlicher Aufgaben die Verfassungsrechtslage und das Menschenbild des Grundgesetzes weitgehend ausgeblendet werden, muss auf eine bemerkenswerte Stellungnahme des Bundesverfassungsgerichts innerhalb des KPD-Urteils hingewiesen werden (BVerfGE $5,85<198>$ ). In dieser hat es zum Sozialstaat unter anderem dargelegt, dass die Tendenz der Ordnung und die in ihr angelegte Möglichkeit der freien Auseinandersetzung zwischen allen realen und geistigen Kräften in Richtung auf Ausgleich und Schonung der Interessen aller wirke. Das Gesamtwohl werde eben nicht von vornherein gleichgestellt mit den Interessen oder Wünschen einer bestimmten Klasse; annähernd gleichmäßige Förderung des Wohles aller Bürger und annähernd gleichmäßige Verteilung der Lasten werde grundsätzlich erstrebt. Es bestehe das Ideal der „sozialen Demokratie in den Formen des Rechtsstaates". Die staatliche Ordnung der freiheitlichen Demokratie müsse demgemäß systematisch auf die Aufgabe der Anpassung und Verbesserung und des sozialen Kompromisses angelegt sein; sie müsse vor allem Missbräuche der Macht hemmen.

\section{VERFASSUNGSRECHTLICHE GRENZEN DER PRIVATISIERUNG}

Die Rechtsprechung des Bundesverfassungsgerichts legt insgesamt die Annahme nahe, dass während der letzten Jahre im Zuge einer groß angelegten Privatisierung öffentlicher Aufgabenfelder verfassungsrechtliche Bindungen missachtet wurden. Das Grundgesetz hat seinen verfassungsrechtlichen "Body-Maß-Index“ in der Regelung des Art. 79 Abs. 3 GG niedergelegt. Hiernach ist eine Änderung des Grundgesetzes, von der die in Art. 1 (Menschenwürde) und Art. 20 (Sozialstaatsprinzip) niedergelegten Grundsätze berührt werden, unzulässig. Man muss gerade im Hinblick auf die geschilderte Entwicklung nachdrücklich darauf hinweisen, dass es sich hierbei um die sogenannte EwigkeitsKlausel handelt.

Wenn schon das Grundgesetz diesbezüglich auch nicht mit qualifizierter Mehrheit geändert werden darf, ist es dem Staat erst recht verwehrt, gleichwirkende Maßnahmen zu ergreifen und das verfassungsrechtlich formell vorgesehene Verfahren zu umgehen. Man kann hierfür das Schlagwort verwenden: „Keine Änderung des Grundgesetzes in seinen tragenden Strukturprinzipien auf kaltem Wege". Mit der zunehmenden Privatisierung öffentlicher Aufgaben hat der Staat jedoch mittlerweile seine eigene Handlungsfähigkeit soweit eingeschränkt, dass er verfassungsrechtlich vorgegebene Ziele nur noch sehr unzureichend erfüllen kann. Daher muss über die Rückgewinnung staatlicher Steuerungsfähigkeit nachgedacht werden.

\section{RÜCKGEWINNUNG STAATLICHER STEUERUNGSFÄHIGKEIT}

Inzwischen ist auch in das Bewusstsein von Politikern und Wirtschaftskreisen die Einsicht vorgedrungen, dass mit der ungebundenen Privatisierung zentraler Bereiche der Daseinsvorsorge ein erheblicher Verlust von Politik- und Steuerungsfähigkeit der Staaten einhergeht. Nicht anders kann man die vielfach erhobene Forderung nach einer Zerschlagung der Macht der Energiekonzerne und nach der Wegnahme ihrer Stromnetze verstehen. Gleiches gilt für die vielfach geäußerte Sorge, ausländische Staatsfonds könnten über Wirtschaftsbeteiligungen nachhaltigen Einfluss auf staatliche Entscheidungen in der Bundesrepublik Deutschland nehmen. Gleichwohl ist immer noch erstaunlich, wie wenig beeindruckt hiervon große Teile der Wissenschaft, wirtschaftswissenschaftlicher Institute und anderer Institutionen wie auch der Medien sind. Folgende Auswege aus 
dem beschriebenen Dilemma bieten sich an:

Zunächst ist der Blick auf Art. 15 GG zu lenken. Hiernach können Grund und Boden, Naturschätze und Produktionsmittel zum Zwecke der Vergesellschaftung durch ein Gesetz, das Art und Ausmaß der Entschädigung regelt, in Gemeineigentum oder in andere Formen der Gemeinwirtschaft überführt werden (Satz 1). Diese Regelung wurde nach Inkrafttreten des Grundgesetzes eher als Trostpflaster für manche politische Gruppierung betrachtet, die man für den Aufbau des demokratischen Rechtsstaats gewinnen wollte. Historisch gesehen und rund 60 Jahre zurückgehend muss man allerdings erkennen, dass die dort genannten Objekte einer Sozialisierung inzwischen aufgrund der modernen technischen und weltweiten wirtschaftlichen Entwicklung in ihrer Bedeutung für unser Staatswesen durch andere abgelöst worden sind. Gleichwohl bleibt die Wertentscheidung des Grundgesetzgebers materiell die gleiche: Sozialisierung etwa von Bodenschätzen würde dann Sinn haben, wenn durch deren missbräuchliche Nutzung durch wenige die innere Unabhängigkeit eines Staatswesens infrage gestellt würde. Das heißt, diese verfassungsrechtliche Befugnis darf nur gebraucht werden, wenn ein Staatswesen in maßgeblichen Teilen handlungsunfähig geworden ist, weil private Unternehmen die staatliche Gewalt inhaltlich zumindest nachhaltig zu steuern vermögen.

In diesem Zusammenhang ist daran zu erinnern, dass zahlreiche Staaten trotz wertvollster Bodenschätze (z. B. Öl oder Metalle) wegen der Tätigkeit ausländischer und international aufgestellter Unternehmen nicht in der Lage sind, eine stabile Gesellschaft mit wirksamem Bildungs- und Sozialsystem zu entwickeln. Nunmehr sind sogar die Grundlagen einer sicheren Ernährung der Menschen in vielen Staaten durch einen ungezügelten Wettbewerb aufgrund einer verfehlten Politik des Internationalen Währungsfonds (IWF), der Welthandelsorganisation (WTO) und der Weltbank gefährdet. Vor diesem Hintergrund ist Art. 15 GG in einem Spannungsverhältnis mit Art. 20 Abs.1 GG und von dieser Strukturnorm zu Art. 79 Abs. 3 und Art. 1 Abs. 1 GG zu sehen.

Es muss deshalb verstärkt darüber nachgedacht werden, viele Privatisierungen im Bereich der Daseinsvorsorge in großen Teilen rückgängig zu machen, oder aber bevorstehende Privatisierungen zu unterlassen. Mit dem zwischen den Koalitionsparteien erzielten Kompromiss für einen Börsengang der Deutschen Bahn muss man jedenfalls eine Bewusstseinsbildung anerkennen, die zumindest ansatzweise in die richtige Richtung geht. Weitere Beispiele für die dringende Aufrechterhaltung der öffentlichen Daseinsvorsorge finden sich im Bereich der Kreditwirtschaft und der Altersvorsorge.

\section{DAS BEISPIEL KREDITWIRTSCHAFT}

Die Bundesrepublik Deutschland muss die Gewährträgerhaftung für Kreditinstitute aufrechterhalten, damit sie weiterhin die Rahmenbedingungen für die Entwicklung von Existenzgründungen und für den Mittelstand bestimmen kann und nicht Teile der deutschen Wirtschaft Opfer undurchsichtiger außerstaatlicher Interessen werden. Es kann nicht von vornherein ausgeschlossen werden, dass infolge der Übernahme großer Kreditinstitute durch ausländische Unternehmen, zum Beispiel auch durch ausländische Staatsfonds, Kreditvergaben an deutsche Unternehmen selbst dann nicht mehr erfolgen, wenn durch Zukunftsinvestitionen in Deutschland Arbeitsplätze in namhafter Zahl neu geschaffen werden können. Sogar die Kreditbedingungen für den Staat würden von außen gesteuert, was man so nicht hinnehmen kann, zumal wenn man den verhängnisvollen Einfluss internationaler RatingAgenturen mit in die Betrachtung einbezieht. Deren Rolle bei der internationalen Bankenkrise wurde überhaupt noch nicht genügend thematisiert und untersucht. Es bedarf schon eines gestörten Verhältnisses zur rechtsstaatlichen freiheitlichen Demokratie westlicher Prägung, wenn man ein Staatswesen Rating-Agenturen und international tätigen Analysten in dem Umfang ausliefert, wie dies seit einigen Jahren der Fall ist. Alle damit verbundenen Wirkungen sind materiell gleichbedeutend mit einer Teilauslieferung der staatlichen Souveränität an nicht demokratisch legitimierte und intransparente Institutionen.

\section{DAS BEISPIEL ALTERSVORSORGE}

In diesem Zusammenhang ist noch auf einen weiteren Bereich der Daseinsvorsorge, der teilweise und im Widerspruch zum Sozialstaatsprinzip des Art. 20 Abs. 1 GG privatisiert worden ist, einzugehen. Es handelt sich um den der Altersvorsorge. Die Forderung des Staates an seine Bürgerinnen und Bürger, zu einem nicht unbeträchtlichen Teil künftig selbst für das Alter vorzusorgen, bedarf näherer Betrachtung. Die Erfahrungen in den USA nach dem Zusammenbruch der New Economy und nunmehr des Immobilienbereichs zeigen, dass dort die Altersversorgung der Menschen in einem erschreckenden Umfang gefährdet ist (Frage: Wo waren hier die Rating-Agenturen und die Analysten?). Zum einen haben auch Weltunternehmen keine Vorsorge für die hieran teilhabenden Betriebsrenten getroffen. Zum andern ist der Verfall der Aktienkurse, zum Teil aufgrund krimineller Machenschaften wie Bilanzmanipulationen, dafür verantwortlich, dass viele Menschen vor dem wirtschaftlichen Ruin und nach Beendigung ihres Arbeitslebens vor dem Nichts stehen. Aus diesem Grunde ist die Forderung, in Eigenverantwortung für die Altersvorsorge tätig zu werden, ohne eine seriöse tragfähige Grundlage inakzeptabel. Letztere muss vom Staat geschaffen werden; das ist seine ureigenste Verantwortung gemäß Art. 20 Abs. 1 Satz 1 GG in Verbindung mit Art. 1 Abs. 1 GG. Erst danach kann diese Forderung an die Menschen gerichtet werden.

\section{FAZIT}

Zur Behebung der beschriebenen Problemlage insgesamt bietet sich eine multifunktionale Strategie an. Eine solche muss zum Ziel haben, die Steuerungsfähigkeit der Staaten zurückzugewinnen und damit einhergehend solide und berechenbare Rahmenbedingungen für den Wirtschaftsstandort Deutschland wie auch für die Altersvorsorge zu schaffen. Darüber hinaus muss diese Strategie darauf ausgerichtet sein, den Arbeitsmarkt wieder in größtmöglichem Umfang dadurch zu stabilisieren, dass die Zahl der regulären Arbeitsverhältnisse deutlich ausgeweitet wird, damit die Sozialkassen einerseits gestärkt und andererseits entlastet werden. Minijobs, Jobsplitting und Mindestlöhne dürfen nicht mehr die Diskussion in diesem Bereich beherrschen. Sie sind Indikatoren für grundlegende Fehlentwicklungen. 